\title{
BMI open Rheumatic heart disease: pilot study for a population-based evaluation of prevalence and cardiovascular outcomes among schoolchildren in Nepal
}

\author{
Nikesh Raj Shrestha, ${ }^{1}$ Bindu Kalesan, ${ }^{2,3}$ Prahlad Karki, ${ }^{1}$ Kunjang Sherpa, ${ }^{1}$ \\ Anil Basnet, ${ }^{1}$ Philip Urban, ${ }^{4}$ Thomas Pilgrim ${ }^{2}$
}

To cite: Shrestha NR, Kalesan B, Karki P, et al. Rheumatic heart disease: pilot study for a populationbased evaluation of prevalence and cardiovascular outcomes among schoolchildren in Nepal. BMJ Open 2012;2: e001616. doi:10.1136/ bmjopen-2012-001616

- Prepublication history for this paper are available online. To view these files please visit the journal online (http://dx.doi.org/10.1136/ bmjopen-2012-001616).

Received 6 June 2012 Accepted 28 September 2012

This final article is available for use under the terms of the Creative Commons Attribution Non-Commercial 2.0 Licence; see http://bmjopen.bmj.com

${ }^{1}$ Department of Internal Medicine and Cardiology, B.P. Koirala Institute of Health Sciences, Dharan, Nepal

${ }^{2}$ Department of Cardiology, Swiss Cardiovascular Center Bern, Bern University Hospital, Bern, Switzerland ${ }^{3}$ Clinical Trials Unit, Department of Social and Preventive Medicine, Bern University, Bern, Switzerland ${ }^{4}$ Department of Cardiology, Hôpital de la Tour, Geneva, Switzerland

\section{Correspondence to} Dr Thomas Pilgrim; thomas.pilgrim@insel.ch

\section{ABSTRACT}

Objectives: To evaluate a protocol for a populationbased programme targeting the prevention of rheumatic heart disease (RHD) progression by early echocardiographic diagnosis of valvular lesions and timely implementation of secondary prevention.

Design: Observational survey with a subsequent prospective cohort study.

Setting: Private boarding school in the urban area of the Sunsari district situated on the foothills of the Lower Himalayan Range in Eastern Nepal.

Participants: Fifty-four unselected school-going children 5-15 years of age, 24 girls and 30 boys.

Primary outcome measure: Logistic feasibility of a large-scale population-based screening study using the echocardiographic criteria formulated by the World Heart Federation, with longitudinal follow-up of children with definite or borderline RHD in a prospective cohort study.

Results: Standardised interview, physical examination and screening echocardiography were performed in a three-staged process and took approximately 6 min per child. Socio-economic status was assessed using surrogate markers such as the occupation of the primary caregiver, numbers of rooms at home, car, television, cell phone and internet connection. Physical examination was focused on cardiac auscultation and signs of acute rheumatic fever and targeted echocardiography was performed by an independent examiner without knowledge of the clinical findings. Two children with evidence of borderline RHD were reexamined at B.P. Koirala Institute of Health Sciences and the indication for secondary antibiotic prevention was discussed with the parents and the children. At 6 months of follow-up, echocardiographic findings were stable in both children. Implementation of secondary antibiotic prevention was challenged by impaired awareness of subclinical RHD among parents and inadequate cooperation with family physicians.

Conclusions: This pilot study shows that the methods outlined in the protocol can be translated into a largescale population-based study. We learned that education and collaboration with teachers, parents and family physicians/paediatricians will be of key importance in order to establish a sustainable programme.

\section{ARTICLE SUMMARY}

Article focus

- Pilot study for a population-based programme targeting prevention of rheumatic heart disease (RHD) progression by early echocardiographic diagnosis of valvular lesions and timely implementation of secondary prevention.

Key messages

- The echocardiographic criteria for diagnosis of RHD provided by the World Heart Federation are a valuable instrument for population-based screening.

- Adequate information of the parents of children with evidence of silent RHD and cooperation with the family physicians is of key importance for long-term adherence to secondary antibiotic prevention.

Strengths and limitations of this study

- The pilot study demonstrates feasibility of a large-scale population-based screening programme for RHD by the use of portable echocardiography, with a subsequent prospective cohort study.

\section{INTRODUCTION}

Rheumatic heart disease (RHD) is estimated to affect more than 15 million people worldwide and leads to 250000 deaths every year. ${ }^{1}$ More than $80 \%$ of the children younger than 15 years of age grow up in regions of the world where RHD is endemic. ${ }^{2}$ A discrepancy of reported prevalence rates of RHD among schoolchildren in developing countries is particularly attributable to diverse sensitivities of echocardiographic and clinical screening, respectively. RHD consolidates a spectrum of different stages of clinically silent and clinically manifest valvular degeneration culminating in congestive heart failure, increasing the risk of endocarditis, cerebrovascular events and eventually leading to 
premature death of the affected working-age population in developing countries.

Acute rheumatic fever (ARF) ensues from an autoimmune reaction in response to group A $\beta$-haemolytic streptococcal pharyngitis and may result in RHD through the cumulative valvular damage of recurrent episodes of $\mathrm{ARF}^{2}$ Whereas overcrowding and poverty are major determinants of disease proliferation, limited access to healthcare resources renders primary prevention with prompt antibiotic treatment of streptococcal pharyngitis difficult to effectuate in large parts of endemic regions of the world. Secondary prevention among children with a documented history of ARF or evidence of RHD is achieved by regular oral or intravenous administration of penicillin continued until early adulthood.

The WHO has recommended echocardiographic screening of RHD in high-prevalence regions. ${ }^{3}$ Early detection of subclinical RHD by use of echocardiography warrants timely implementation of secondary antibiotic prophylaxis and may prevent progression of valvular damage leading to congestive heart failure. We performed a pilot study in order to test the feasibility of a cross-sectional survey and a longitudinal cohort study to assess prevalence and clinical outcome of RHD according to the World Heart Federation criteria. ${ }^{4}$

\section{AIMS AND OBJECTIVES}

The goal of the project consists in the prevention of RHD progression by early echocardiographic diagnosis of valvular lesions and timely implementation of secondary prevention. We designed a study to investigate the prevalence rate of definite and borderline RHD among children in Eastern Nepal, and to assess long-term clinical outcome of children undergoing secondary antibiotic prevention for RHD. ${ }^{5}$ The objective of the pilot study was to evaluate the administrative and logistic feasibility of a large-scale population-based screening study, specifically pertaining to questionnaire design and protocol adherence, and to assess the recruitment of children with signs of RHD into a prospective cohort with longitudinal follow-up.

\section{METHODS}

Study design and setting

The design of the study has been outlined in detail previously. ${ }^{5}$ In summary, a cross-sectional survey of schoolchildren aged 5-15 years will be performed for clinically silent or manifest RHD in the Sunsari district of Eastern Nepal. The location in rural or urban areas, as well as public or private administration of the schools will be used as a surrogate to reflect the socio-economic demographic distribution of the population in Eastern Nepal. Children found to have signs consistent with RHD will be enrolled in a prospective cohort to be followed over the course of 5 years. The Sunsari district is situated on the foothills of the Lower Himalayan Range in Eastern
Nepal and extends to the south of the city of Dharan, the third-largest city in Nepal.

\section{Study population}

The selection of the school for the pilot study was driven by practical reasons and was not based on a prespecified sampling procedure to obtain a representative study population. As a consequence, the cohort of the pilot study is not eligible for inclusion into the actual study cohort.

A small private boarding school in the urban area of the Sunsari district was selected. A discussion with the principal of the school was performed in order to outline the aim of the project and obtain informed consent. All children of this particular school underwent screening echocardiography.

\section{Data collection}

Data acquisition was organised in a three-staged process by study nurses and physicians. School teachers organised the transfer of the children between the study nurses and physicians gathering demographic characteristics, performing physical examination and conducting echocardiography, respectively. Data on social background and medical history were acquired in a standardised interview on the basis of a questionnaire. Demographic variables such as age, household characteristics and socio-economic indicators were recorded along with a short medical history followed by physical examination documenting height, weight and potential clinical signs of ARF. Study nurses questioned the children about demographic characteristics, filled in the questionnaire, and measured height and weight. A first physician completed the medical history and executed physical examination including cardiac auscultation. A second independent physician performed screening echocardiography using the Samsung portable U6 echocardiography machine to document morphological and/or functional valvular lesions consistent with RHD.

Parents of children with evidence of RHD were contacted by the physicians and oriented about the findings. The children and parents were subsequently invited for a thorough examination at B.P. Koirala Institute of Health Sciences. Follow-up data of children with borderline or definite RHD was collected during a dedicated follow-up visit 6 months after screening.

All data were documented on dedicated paper case report forms that were subsequently transferred into an electronic database.

\section{Definitions}

RHD was classified in accordance with the WHF criteria for individuals aged $\leq 20$ years into definite and borderline. ${ }^{4}$ Definite RHD was divided into four subcategories. Subcategory A is defined by pathological mitral regurgitation and at least two morphological features of RHD of the mitral valve, subcategory $B$ is diagnosed in the presence of mitral stenosis with a mean gradient of $\geq 4 \mathrm{~mm} \mathrm{Hg}$. 
Subcategory C is characterised by pathological aortic regurgitation in combination with at least two morphological features of RHD of the aortic valve, and subcategory $\mathrm{D}$ is determined by borderline disease of both the aortic valve and the mitral valve. Borderline RHD is partitioned into three subcategories. Subcategory A is determined by the presence of at least two morphological features of RHD of the mitral valve without pathological mitral regurgitation or mitral stenosis, subcategories B and $\mathrm{C}$ are characterised by pathological mitral regurgitation, or pathological aortic regurgitation, respectively. Physiological mitral regurgitation (A), physiological aortic regurgitation (B) and an isolated morphological feature of RHD of the mitral or aortic valve (ie, valvular thickening) without any associated pathological stenosis or regurgitation $(\mathrm{C}$ and $\mathrm{D}$, respectively) are classified as normal echocardiographic findings.

\section{Ethics and funding}

The study was conducted in compliance with the declaration of Helsinki and was registered with clinical trials.gov (NCT01550068). The study was approved by the ethic committee of B.P. Koirala Institute of Health Sciences and was given an exempt status by the ethics committee at University of Bern, Switzerland (KEK-BE 018/12). The pilot study was supported by an unrestricted grant from the foundation 'Coeur de la Tour' (http://www. coeurdelatour.ch) from Geneva, Switzerland.

\section{Sample size calculation}

Sample size was determined by practical aspects rather than estimations of prevalence rates. In order to adequately evaluate the questionnaires and assess the duration of a screening examination, we considered a mixed cohort of schoolchildren across all age groups important. We considerably selected a private school with a small number of students in order to allow screening of all children from the selected school in a single day. The localisation of the school in an urban area of the lower-middle class with only limited access to electricity throughout the hours of the day simulated a challenge often encountered in more rural areas of Eastern Nepal. The screening examinations were timed and performed in a single afternoon.

\section{Statistical analysis}

Statistical analyses were performed using SPSS Statistics V.17.0. Continuous variables are presented as mean \pm SD. Categorical data are expressed as frequency (percentages).

\section{RESULTS}

\section{Observational survey}

Fifty-four children from a private boarding school in the urban area of the Sunsari district ranging from 5 to 15 years of age were included in the pilot observational survey, which was performed by two cardiologists, a paediatrician, an internal medicine resident and two nurses. Screening was scheduled for a single afternoon on a regular school day. Standardised interview, physical examination and screening echocardiography were performed in a three-staged process and took all together approximately 6 min per child. Screening examinations for this pilot study were performed in two dedicated rooms, one for interview and physical examinations and another one for echocardiography. Boys and girls were examined separately; cardiac auscultation of girls was performed by a female or a male doctor in the presence of a female chaperone. Teachers ensured the efficient realisation of the screening process and engaged children waiting to be examined.

Socio-economic status was evaluated using specific questions addressing surrogate markers such as the occupation of the primary caregiver, numbers of rooms at home, car, television, cell phone and internet connection. These questions were readily answered by the majority of the children irrespective of age. Most of the children were belonging to lower middle-class families, where the primary caregiver was a manual worker, five family members were living in three rooms, owned a television and cell phone, but had no car and no internet connection at home (table 1). In turn, questions pertaining to a documented history of a previous attack of rheumatic fever had to be addressed using approximative closed questions about regular medication intake, hospitalisations or prolonged disease in the past, and joint pain. No verification with parents or medical documentation was performed.

Physical examination documented height and weight, and was focused on cardiac auscultation and signs of acute rheumatic fever. Mean age was 9.6 2 .6 years, and $56 \%$ of the children were boys. Cardiac auscultation revealed four children with soft systolic heart murmurs.

Targeted echocardiography was performed by an independent examiner without knowledge of the clinical

\begin{tabular}{lc} 
Table $1 \quad$ Pilot study baseline characteristics \\
\hline Baseline characteristics & $\mathbf{n}(\%)$ or mean (SD) \\
\hline Age (years) & $9.6(2.9)$ \\
Height in $(\mathrm{cm})$ & $127(16)$ \\
Weight in $(\mathrm{kg})$ & $28(12)$ \\
Male & $30(55.6)$ \\
Documented history of ARF & $0(0)$ \\
Socio-economic status & \\
$\quad$ Number of family members & $4.9(1.2)$ \\
$\quad$ Children & $2.2(0.9)$ \\
$\quad$ Adults & $2.6(1.0)$ \\
Amenities at home & \\
Household, Number of rooms & $2.8(1.6)$ \\
Family owns a car & $1(1.9)$ \\
Television at home & $50(92.6)$ \\
Internet connection at home & $4(7.7)$ \\
Family uses a cell phone & $51(94.4)$ \\
\hline
\end{tabular}


findings. While none of the children had echocardiographic evidence of definite RHD, two children were found to have borderline RHD, and two children had isolated thickening of the anterior mitral valve leaflet without pathological regurgitation, which did not meet the criteria for borderline RHD according to the WHF criteria. A 12-year-old asymptomatic boy with no history of ARF and a soft systolic heart murmur was found to have significant thickening of the anterior mitral valve leaflet associated with pathological mitral regurgitation and mild tricuspid regurgitation. Another asymptomatic 12-year-old boy with a soft systolic heart murmur had restricted posterior mitral valve leaflet motion with evidence of pathological mitral regurgitation and mild tricuspid regurgitation. During a subsequent outpatient visit at B.P. Koirala Institute of Health Sciences, the indication for secondary antibiotic prevention was discussed with the parents and the children, and orientation for inclusion into a prospective registry was provided.

Barriers to implementation of the observational survey included limited electricity supply around the clock and unpredictable power cuts rendering a battery-operated portable echocardiography machine indispensable.

The selection process of schools that adequately reflect the demographic distribution of the target area was outlined previously and was not assessed in the present pilot study. ${ }^{5}$

\section{Longitudinal follow-up}

Both children with evidence of borderline RHD are being prospectively followed up and have been invited for a clinical and echocardiographic follow-up examination at 6 months after screening. None of the children had experienced an adverse event and echocardiographic findings were stable as compared with baseline in both kids.

Secondary antibiotic prevention was discontinued in both children within the first 6 months of follow-up. The family physicians who had not been involved in the initial decision for initiation of prevention had recommended against prolonged antibiotic treatment in the absence of pathological heart murmurs and no documented attacks of rheumatic fever.

Implementation of secondary antibiotic prevention was challenged by impaired understanding of subclinical RHD among parents and family physicians/paediatricians. Limited public awareness and education, as well as inadequate collaboration with family physicians emerged as barriers to compliance with secondary prevention.

\section{DISCUSSION}

We report the results of a pilot study evaluating a research protocol for an observational survey of RHD among school-going children and a subsequent prospective cohort study of children with evidence of rheumatic valvular lesions. This pilot study represents an integral part for the final implementation of the protocol in a large population-based setting. The main findings can be summarised as follows:

1. Clinical and echocardiographic screening of RHD is feasible within 5-10 min per child. Active involvement of the teachers who are familiar with the children not only facilitates the organisation but also anticipates distrust towards medical personnel from both children and teachers.

2. Adequate orientation of and collaboration with the parents after diagnosis of RHD in a child is of key importance to adherence to preventive antibiotic treatment. Accepting the diagnosis of subclinical RHD in an apparently healthy child is difficult for both the parents and the children and calls for continued support.

3. Collaboration with family physicians and paediatricians is important to improve adherence to secondary prevention for clinically silent RHD. Inconsistent information causes insecurity and decreases the motivation for compliance with regular antibiotic prevention.

4. The WHF criteria are a helpful instrument to classify subclinical valvular lesions consistent with RHD.

We learned from this pilot study, that collaboration with teachers, parents and family physicians/pasediatricians is indispensable to perform efficient screening, improve compliance with secondary prevention and ascertain clinical follow-up.

Informed teachers were allies in our study not only in terms of facilitating the organisation of the pilot study, but also with regard to the orientation of the parents of the two children diagnosed with clinically silent RHD. Moreover, completion of parts of the questionnaire asking for socio-economic background could be assisted by the teachers and would improve quality of the data. As a consequence we plan to extend the participation of the teachers in the collection of the baseline criteria in the main study.

In addition to the school teachers, the parents of the children will be the key players for successful completion of the programme. Accepting disease in an asymptomatic child is difficult for every parent. Continued education during every clinical visit with adequate cultural sensitivity should reinforce adherence to treatment. Given the high illiteracy rate in Nepal, information brochures distributed to the parents will not achieve the targeted objective. In response to the need for education on RHD diagnosis and prevention, we are therefore preparing a documentary film that will be broadcasted on local television and in schools before the screening examinations begin.

Collaboration with family physicians and paediatricians will be central to improve long-term adherence to secondary antibiotic prevention for silent RHD. Inconsistent information will cause insecurity among parents and children and decrease the motivation for compliance with regular antibiotic prevention. Continuing education will be provided to 
family physicians in the area in order to enhance collaboration and coordinate recommendations on secondary prevention.

Previous studies using primary screening echocardiography performed in endemic regions in Southeast Asia, ${ }^{6-8}$ the Western Pacific, ${ }^{9}{ }^{10}$ Africa $^{6}{ }^{11}$ and Central America $^{12}$ reported prevalence rates of RHD among children ranging from $20.4 / 1000$ to 55.2/000 children most of which using the $2006 \mathrm{WHO}$ criteria. In contrast to the WHO criteria combining both, clinical and echocardiographic findings, the WHF criteria are based on echocardiographic criteria only, and have been applied for the present pilot study. ${ }^{3}$ Studies documenting echocardiographic follow-up reported stable disease or even regression of valvular lesions in three quarters of the children, but were mainly limited by a short duration of follow-up and a small sample size. ${ }^{7} 812$

The recently released WHF criteria for echocardiographic diagnosis of RHD proved helpful in the classification of valvular lesions related to subclinical RHD and provide an adequate instrument for large populationbased studies.

Acknowledgements We would like to thank Sina Pilgrim, MD; Kavita Shah, RN and Kishori Dhakal, RN.

Contributors NS, BK and TP substantially contributed to the conception and design, drafting the article and final approval of the version to be published. $\mathrm{PK}, \mathrm{KS}, \mathrm{AB}$ and PU substantially contributed to the conception and design, revising the content of the draft and final approval of the version to be published.

Funding The study is supported by an unrestricted grant from the foundation 'Coeur de la Tour' (http://www.coeurdelatour.ch) from Geneva, Switzerland.

Competing interests None.

Patient consent Obtained.

Ethics approval Ethics Committee of Bern University Hospital; Institutional Review Board of B.P. Koirala Institute of Health Sciences.
Provenance and peer review Not commissioned; externally peer reviewed.

Data sharing statement There are no additional data available.

\section{REFERENCES}

1. Carapetis JR, Steer AC, Mulholland EK, et al. The global burden of group a streptococcal diseases. Lancet Infect Dis 2005;5:685-94.

2. Marijon E, Mirabel M, Celermaier DS, et al. Rheumatic heart disease. Lancet 2012;379:953-64.

3. Carapetis J, Parr J, Cherian T. Standartization of epidemiologic protocols for surveillance of post-streptococcal sequelae: acute rheumatic fever, rheumatic heart disease and acute post-streptococcal glomerulonephritis. Department of Health and Human Services, National Institutes of Health (online). 2010. http://www.niaid.nih.gov/ topics/strepThroat/Documents/groupasequelae.pdf (accessed 4 June 2012)

4. Remenyi B, Wilson N, Steer A, et al. World Heart Federation criteria for echocardiographic diagnosis of rheumatic heart disease-an evidence-based guideline. Nat Rev Cardiol 2012;9:297-309.

5. Pilgrim T, Kalesan B, Karki P, et al. Protocol for a population-based study of rheumatic heart disease prevalence and cardiovascular outcomes among schoolchildren in Nepal. BMJ Open 2012;2 e001320. doi:10.1136/bmjopen-2012-001320.

6. Marijon E, Ou P, Celermajer DS, et al. Prevalence of rheumatic heart disease detected by echocardiographic screening. $N$ Engl J Med 2007;357:470-6.

7. Bhaya M, Panwar S, Beniwal R, et al. High prevalence of rheumatic heart disease detected by echocardiography in school children. Echocardiography 2009;27:448-53.

8. Saxena A, Ramakrishnan S, Roy A, et al. Prevalence and outcome of subclinical rheumatic heart disease in India: The RHEUMATIC (Rheumatic Heart Echo Utilization and Monitoring Actuarial Trends in Indian Children) study. Heart 2011;97:2018-22.

9. Reeves BM, Kado J, Brook M. High prevalence of rheumatic heart disease in Fiji detected by echocardiography screening. J Paediatr Child Health 2011;47:473-8.

10. Webb RH, Wilson NJ, Lennon DR, et al. Optimising echocardiographic screening for rheumatic heart disease in New Zealand: not all valve disease is rheumatic. Cardiol Young 2011;21:436-43.

11. Beaton A, Okello E, Lwabi $P$, et al. Echocardiography screening for rheumatic heart disease in Ugandan schoolchildren. Circulation 2012;125:3127-32.

12. Paar JA, Berrios NM, Rose JD, et al. Prevalence of rheumatic heart disease in children and young adults in Nicaragua. Am J Cardiol 2010;105:1809-14. 